\title{
ON THE GOLDBACH CONJECTURE IN ARITHMETIC PROGRESSIONS
}

\author{
CLAUS BAUER AND WANG YONGHUI
}

\begin{abstract}
It is proved that for a given integer $N$ and for all but $\ll(\log N)^{B}$ prime numbers $k \leq N^{5 / 48-\varepsilon}$ the following is true: For any positive integers $b_{i}, i \in\{1,2,3\},\left(b_{i}, k\right)=1$ that satisfy $N \equiv b_{1}+b_{2}+b_{3}(\bmod k), N$ can be written as $N=p_{1}+p_{2}+p_{3}$, where the $p_{i}, i \in\{1,2,3\}$ are prime numbers that satisfy $p_{i} \equiv b_{i}(\bmod k)$.
\end{abstract}

1. Introduction. Vinogradov $[\mathbf{1 7}]$ has proved that every sufficiently large odd positive integer can be written as the sum of three primes. This theorem has been generalized in many ways. In 1953, Ayoub [1] proved the following result: If $k$ is a fixed positive integer, $b_{i}, i=1,2,3$, are integers with $\left(b_{i}, k\right)=1$ and $J\left(N ; k, b_{1}, b_{2}, b_{3}\right)$ is the number of solutions of the equation

$$
\left\{\begin{array}{l}
N=p_{1}+p_{2}+p_{3} \\
p_{j} \equiv b_{j}(\bmod k)
\end{array}\right.
$$

then

$$
J\left(N ; k, b_{1}, b_{2}, b_{3}\right)=(N ; k) \frac{N^{2}}{2 \log ^{3} N}(1+o(1)),
$$

where for odd integer $N \equiv b_{1}+b_{2}+b_{3}(\bmod k)$,

$$
\begin{aligned}
\sigma(N, k)= & \frac{C(k)}{k^{2}} \prod_{p \mid k} \frac{p^{3}}{(p-1)^{3}+1} \prod_{\substack{p \mid N \\
p \nmid k}} \frac{(p-1)\left((p-1)^{2}-1\right)}{(p-1)^{3}+1} \\
& \times \prod_{p>2}\left(1+\frac{1}{(p-1)^{3}}\right),
\end{aligned}
$$

2000 AMS Mathematics Subject Classification. Primary 11P32, 11L07.

The work of the second author was supported partly by Chinese NSF Tianyuan Youth Foundation (10126001) and was done while he was a visiting scholar at the Morningside Center.

Received by the editors on January 6, 2003, and in revised form on July 22, 2004 . 
where all $p>2, C(k)=2$ for odd $k$ and $C(k)=8$ for even $k$.

Using Ayoub's method, one can prove this result for all $k \leq \log ^{A} N$ for an arbitrary $A>0$ for all sufficiently large odd integers $N$. Liu and Zhan $[\mathbf{1 1}]$ as well as the first author [2] improved upon Ayoub's result by proving the following theorem:

For $N \equiv b_{1}+b_{2}+b_{3}(\bmod k)$ and an odd $N$ sufficiently large, there holds

$$
J\left(N ; k, b_{1}, b_{2}, b_{3}\right)>0
$$

for all $k \leq N^{\delta}$, where $\delta$ is a very small, positive constant.

In [10], it was shown that (1.1) holds for all $k \leq R=N^{(1 / 8)-\varepsilon}$ with at most $\ll R(\log N)^{-A}$ exceptions for any $A>0$. Liu proved in [7] that if $k$ is restricted to be a prime number, $R$ can be chosen as large as $N^{3 / 20}(\log N)^{-A}$ for any $A>0$. Here we give a result that improves on the result in $[7]$ by obtaining a significantly smaller set of exceptional modules $k$ at the cost of a smaller upper bound $R$ :

Theorem 1. Let $R=N^{5 / 48-\varepsilon}$. Then the inequality (1.1) holds for all prime numbers $k \leq R$ with at most $O\left((\log N)^{B}\right)$ exceptions for a certain $B>0$.

The improvement in this paper compared to previous work is due to two innovations. First, we apply a technique previously used in [9] to our problem. Second, as a main contribution of our paper, we exactly calculate the contribution of $N$-exceptional zeros that we define in the following. We set

$$
L=\log N, \quad L_{2}=\log \log N, \quad L(s, \chi)=\sum_{n \geq 1} \frac{\chi(n)}{n^{s}},
$$

where $\chi$ is a Dirichlet character. For a prime number $k, k \leq N$, and a fixed positive integer $V$, we define

$$
\begin{gathered}
P_{k}=\{m \in \mathbf{N}: m \equiv 0(\bmod k)\}, \quad I_{V}=\left[k, k L^{V}\right] \bigcup\left[k^{2}, k^{2} L^{V}\right], \\
A_{k}=P_{k} \cap I_{V} .
\end{gathered}
$$


We call a Dirichlet character $\chi$ to a module $q, q \leq N$, an $N$-exceptional character if there exists at least one complex number $s=\sigma+i t$ such that

$$
\sigma>1-\frac{E L_{2}}{L}, \quad|t| \leq N, \quad L(s, \chi)=0,
$$

where $E$ is a fixed, positive number to be defined later. We call $s$ an $N$-exceptional zero and we call an integer $q$ an $N$-exceptional integer if there exists an $N$-exceptional character $\chi$ modulo $q$.

We note that the concept of $N$-exceptional zeros has earlier been applied to other problems in additive prime number theory in $[\mathbf{1 8}]$ and [3]. However, the exact definitions of the $N$-exceptional zeros in both papers differ from the definition given here and, indeed, the sets of $N$-exceptional zeros defined here and in $[\mathbf{1 8}]$ and $[\mathbf{3}]$ have no common elements.

Theorem 1 is a direct consequence of Theorems 2 and 3 .

Theorem 2. For a given prime number $k \leq N^{5 / 48-\varepsilon}$, if none of the integers $q \in A_{k}$ is $N$-exceptional, then (1.1) is true for this $k$.

Theorem 3. There are at most $O\left((\log N)^{B}\right)$ prime numbers $k$, $1 \leq k \leq N$, such that at least one of the integers $q \in A_{k}$ is $N$ exceptional. Here, $B$ is a fixed positive constant.

\section{Outline of the proof of Theorem 2 and treatment of the} minor arcs. In the sequel, $\left[a_{1}, \ldots, a_{n}\right]$ denotes the least common multiple of the integers $a_{1}, \ldots, a_{n} . c$ is an effective positive constant and $\varepsilon$ will denote an arbitrarily small positive number; both of them may take different values at different occasions. For example, we may write

$$
L^{c} L^{c} \ll L^{c}, \quad N^{\varepsilon} L^{c} \ll N^{\varepsilon} .
$$

We use the familiar notations

$$
\sum_{\chi \bmod q} *:=\sum_{\substack{\chi \bmod q \\ \chi \text { primitive }}}, \quad \sum_{1 \leq a \leq q}^{*}:=\sum_{\substack{1 \leq a \leq q \\(a, q)=1}} .
$$


We know from [1] that Theorem 2 holds true for $k \leq L^{H}$ for any $H>0$. Therefore, we assume throughout the document that

$$
k>L^{H}
$$

for a fixed $H>0$ to be determined later. $\chi_{q}$ denotes a character modulo $q$ and $\chi_{q, 0}$ is the principal character modulo $q$. We write $e(\alpha)=e^{2 \pi i \alpha}$ and the variables $p$ and $k$ always denote prime numbers. We keep $k$ fixed throughout this paper. If $p^{m} \mid q$, but $p^{m+1} \nmid q$, we write $p^{m} \| q$. We define for any three positive integers $r_{i}, i \in\{1,2,3\}$ that satisfy $k^{3} \nmid r_{i}$,

$$
s_{i}= \begin{cases}r_{i} & \text { if } k \nmid r_{i}, \\ r_{i} / k & \text { if } k \| r_{i}, \\ r_{i} / k^{2} & \text { if } k^{2} \| r_{i}\end{cases}
$$

Setting $\left[r_{1}, r_{2}, r_{3}\right]=r$ and $\left[s_{1}, s_{2}, s_{3}\right]=s$, this implies for $k^{m} \| r, m \leq 2$ :

$$
r=s k^{m} \text {. }
$$

For a positive integer $q$ and a character $\chi$ modulo $q$, let

$$
\begin{gathered}
k_{q}=(k, q), \quad R(N)=\sum_{\substack{N / 4 \leq n_{i}<N \\
n \equiv b_{i}(\bmod k) \\
n_{1}+n_{2}+n_{3}=N}} \Lambda\left(n_{1}\right) \Lambda\left(n_{2}\right) \Lambda\left(n_{3}\right), \\
C(\chi, q, h, b, a)=\sum_{\substack{m=1 \\
m(\bmod h)}}^{q} \chi(m) e\left(\frac{m a}{q}\right), \\
\quad \times \frac{1}{\phi^{3}(q)} \sum_{\substack{a=1 \\
(a, q)=1}}^{q} C\left(\chi_{1}, q, k_{q}, b_{1}, a\right) C\left(\chi_{2}, q, k_{q}, b_{2}, a\right) \\
\left.\left.k_{q}, \chi_{1}, \chi_{2}, \chi_{3}\right): q, k_{q}, b_{3}, a\right) e\left(\frac{-a N}{q}\right) \\
A\left(N, q, k_{q}\right)=Z\left(N, q, k_{q}, \chi_{\left(q / k_{q}\right), 0}, \chi_{\left(q / k_{q}\right), 0}, \chi_{\left(q / k_{q}\right), 0}\right) \\
\sum_{N / 4<n \leq N} e(\lambda n) .
\end{gathered}
$$


As we always argue for fixed variables $N$ and $k$, denote by

$$
\begin{gathered}
S\left(\lambda, b_{i}\right)=\sum_{\substack{N / 4<n \leq N \\
n \equiv b_{i}(\bmod k)}} \Lambda(n) e(n \lambda), \quad S(\lambda, \chi)=\sum_{N / 4<n \leq N} \Lambda(n) e(n \lambda) \chi(n), \\
W(\lambda, \chi)=S(\lambda, \chi)-E_{0}(\chi) T(\lambda), \quad E_{0}(\chi)= \begin{cases}1 & \text { if } \chi=\chi_{0}, \\
0 & \text { otherwise, }\end{cases} \\
P_{1}=k^{4 / 3} L^{3 G}, \quad P_{2}=k^{2} L^{3 G}, \quad Q=N k^{-2} L^{-4 G},
\end{gathered}
$$

where the constant $G \geq 8$ will be specified later. Using the circle method, we define the major $\operatorname{arcs} M=E_{1}(k) \cup E_{2}(k)$ as in [7]:

$$
\begin{aligned}
& E_{1}(k)=\bigcup_{\substack{q \leq P_{1} \\
k \nmid q}} \bigcup_{\substack{a=1 \\
(a, q)=1}}^{q}\left[\frac{a}{q}-\frac{1}{q Q}, \frac{a}{q}+\frac{1}{q Q}\right], \\
& E_{2}(k)=\bigcup_{\substack{q \leq P_{2} \\
k \mid q}} \bigcup_{\substack{a=1 \\
(a, q)=1}}^{q}\left[\frac{a}{q}-\frac{1}{q Q}, \frac{a}{q}+\frac{1}{q Q}\right],
\end{aligned}
$$

We define the minor $\operatorname{arcs} m$ as $m=[(1 / Q), 1+(1 / Q)] \backslash M$. Writing $\alpha=(a / q)+\lambda$, we use Dirichlet's theorem on rational approximation and find that $m \subset E_{3}(k) \cup E_{4}(k)$, where

$$
\begin{aligned}
& E_{3}(k)=\left\{\alpha=\frac{a}{q}+\lambda: P_{1}<q<Q, k \nmid q,|\lambda| \leq \frac{1}{q Q}\right\}, \\
& E_{4}(k)=\left\{\alpha=\frac{a}{q}+\lambda: P_{2}<q<Q, k|q,| \lambda \mid \leq \frac{1}{q Q}\right\} .
\end{aligned}
$$

We see

$$
\begin{aligned}
R(N)= & \int_{1 / Q}^{1+(1 / Q)} e(-N \alpha) \prod_{i=1}^{3} S\left(\alpha, b_{i}\right) d \alpha \\
= & \left(\sum_{i=1}^{2} \int_{E_{i}(k)}\right) e(-N \alpha) \prod_{i=1}^{3} S\left(\alpha, b_{i}\right) d \alpha \\
& +O\left(\sum_{i=3}^{4} \int_{E_{i}(k)}\left|\prod_{i=1}^{3} S\left(\alpha, b_{i}\right)\right| d \alpha\right) \\
= & : R_{1}(N)+R_{2}(N)+O\left(R_{3}(N)+R_{4}(N)\right) .
\end{aligned}
$$


To estimate the contribution of the integral over $m$, we quote the following lemma from $[\mathbf{7}]$ :

Lemma 2.1. Let $A>0$ be arbitrary and $\alpha \in E_{3}(k) \cup E_{4}(k)$. If in (2.4) $G=G(A)$ is chosen sufficiently large, then

$$
S(\alpha, b) \ll \frac{N}{k L^{A+1}} .
$$

We derive from Lemma 2.1 and Dirichlet's lemma on rational approximation the following estimate:

$$
\begin{aligned}
\int_{E_{3}(k) \cup E_{k}(4)} \mid & S\left(\alpha, b_{1}\right) S\left(\alpha, b_{2}\right) S\left(\alpha, b_{3}\right) \mid d \alpha \\
\ll & \max _{\alpha \in E_{3}(k) \cup E_{4}(k)}\left|S\left(\alpha, b_{1}\right)\right|\left(\int_{0}^{1}\left|S\left(\alpha, b_{2}\right)\right|^{2} d \alpha\right)^{1 / 2} \\
& \times\left(\int_{0}^{1}\left|S\left(\alpha, b_{3}\right)\right|^{2} d \alpha\right)^{1 / 2} \\
\ll & \frac{N^{2}}{k^{2} L^{A}} .
\end{aligned}
$$

In the following sections, we shall show that, under the condition of Theorem 2,

$$
R_{1}(N)+R_{2}(N)=\sigma(N, k) \frac{N^{2}}{32}+O\left(N^{2} k^{-2} L^{-A}\right),
$$

for any $A>0$ and where $\sigma(N, k)$ is defined as in (1.2). Using

$$
\frac{k}{\phi^{3}(k)} \gg \sigma(N, k) \gg \frac{k}{\phi^{3}(k)},
$$

Theorem 2 follows from (2.5), (2.6) and (2.7).

\section{Preliminary lemmas.}

Lemma 3.1. Let $f(x), g(x)$ and $f^{\prime}(x)$ be three real differentiable and monotonic functions in the interval $[a, b]$ and $|g(x)| \ll M$. 
(i) If $\left|f^{\prime}(x)\right| \gg m>0$, then

$$
\int_{a}^{b} g(x) e(f(x)) d x \ll M / m .
$$

(ii) If $\left|f^{\prime \prime}(x)\right| \gg r>0$, then

$$
\int_{a}^{b} g(x) e(f(x)) d x \ll M / r^{1 / 2}
$$

Proof. See [13, Chapter 21].

Lemma 3.2. For any natural number $q=q_{1} q_{2},\left(q_{1}, q_{2}\right)=1$ and characters $\chi_{a}(\bmod q)=\chi_{a_{1}}\left(\bmod q_{1}\right), \chi_{a_{2}}\left(\bmod q_{2}\right), \chi_{b}(\bmod q)=$ $\chi_{b_{1}}\left(\bmod q_{1}\right) \chi_{b_{2}}\left(\bmod q_{2}\right), \chi_{c}(\bmod q)=\chi_{c_{1}}\left(\bmod q_{1}\right), \chi_{c_{2}}\left(\bmod q_{2}\right)$ and $f=f_{1} q_{2}+f_{2} q_{1}$, there is:

a) $C\left(\chi_{a}, q, k_{q}, b, f\right)=C\left(\chi_{a_{1}}, q_{1}, k_{q_{1}}, b, f_{1}\right) C\left(\chi_{a_{2}}, q_{2}, k_{q_{2}}, b, f_{2}\right)$,

b) $Z\left(N, q, k_{q}, \chi_{a}, \chi_{b}, \chi_{c}\right)=Z\left(N, q_{1}, k_{q_{1}}, \chi_{a_{1}}, \chi_{b_{1}}, \chi_{c_{1}}\right) Z\left(N, q_{2}, k_{q_{2}}\right.$, $\left.\chi_{a_{2}}, \chi_{b_{2}}, \chi_{c_{2}}\right)$.

c) If $\chi$ modulo $p^{\beta}$ is a both nonprimitive and nonprincipal character, i.e., $\chi$ is induced by $\chi^{*}$ modulo $p^{\alpha}, 1 \leq \alpha<\beta$, then for $(b, p)=1$, $(a, p)=1$ and $0 \leq \gamma<\beta$, we have

$$
C\left(\chi, p^{\beta}, p^{\gamma}, b, a\right)=0
$$

Proof. Parts a) and b) are shown in the same way as Lemma 4.4 a and b in [2]. Part c) is Lemma 4.3 in [2].

Lemma 3.3. Set $(a, q)=1$ and $(b, q)=1$ throughout the lemmata a) and b).

a) Let $\chi$ be a character modulo $q$. Then

$$
C(\chi, q, 1, b, a) \ll q^{1 / 2} .
$$


b)

$$
\begin{aligned}
& C\left(\chi_{q, 0}, q, k_{q}, b, a\right) \\
& \quad= \begin{cases}\mu\left(q / k_{q}\right) e\left(t b a / k_{q}\right) & \text { if }\left(q / k_{q}, k_{q}\right)=1, t q / k_{q} \equiv 1\left(\bmod k_{q}\right), \\
0 & \text { otherwise. }\end{cases}
\end{aligned}
$$

c) Let there be given any three characters $\chi_{1}, \chi_{2}, \chi_{3}$, modulo $k^{2}$. Then

$$
Z\left(N, k^{2}, k, \chi_{1}, \chi_{2}, \chi_{3}\right) \neq 0 \Longrightarrow \chi_{1}, \chi_{2}, \chi_{3}
$$

are primitive characters modulo $k^{2}$.

d) For any three primitive characters $\chi_{i}$ modulo $r_{i}, 1 \leq i \leq 3$ with $k^{2} \| r$ where $\left[r_{1}, r_{2}, r_{3}\right]=r, r \mid q$, and the principal character $\chi_{0}$ modulo $q$ we have:

$$
Z\left(N, q, k, \chi_{1} \chi_{0}, \chi_{2} \chi_{0}, \chi_{3} \chi_{0}\right) \neq 0 \Longrightarrow k^{2} \| r_{i}, \quad 1 \leq i \leq 3
$$

e) For any $\chi_{1}, \chi_{2}, \chi_{3}$ modulo $k^{2}$

$$
Z\left(N, k^{2}, k, \chi_{1}, \chi_{2}, \chi_{3}\right) \ll k^{-2}
$$

Proof. Part a) is contained in Lemmas 5.1 and 5.2 in [12]. Part b) is shown in $[\mathbf{1 6}]$.

c) If any $\chi_{i}=\chi_{0}\left(\bmod k^{2}\right), 1 \leq i \leq 3$, then the lemma follows from Lemma $3.3 \mathrm{~b}$ ). If any of $\chi_{i}$ is a nonprimitive character modulo $k^{2}$ that is induced by a primitive character modulo $k$, then the lemma follows from Lemma $3.2 \mathrm{c})$.

d) Applying Lemma $3.2 \mathrm{~b})$, we can write $Z(N, q, k, \ldots)=Z\left(N, r^{\prime}, k\right.$, $\ldots$ ) $A(N, l, 1)$, where $\left(r^{\prime}, l\right)=1, r \mid r^{\prime}$, and every prime factor that divides $r^{\prime}$ also divides $r$. From Lemma $\left.3.2 \mathrm{c}\right)$, we see that $Z\left(N, r^{\prime}, k, \ldots\right)=$ 0 , if $r^{\prime} \neq r$. Using the notation introduced in (2.3) and again Lemma $3.2 \mathrm{~b})$, we find $Z(N, r, k, \ldots)=Z(N, s, 1, \ldots) Z\left(N, k^{2}, k, \ldots\right)$. Thus, the proof can focus on terms $Z(N, q, \ldots)$ that can be written as $Z(N, q, k, \ldots)=Z(N, s, 1, \ldots) Z\left(N, k^{2}, k, \ldots\right) A(N, l, 1)$, where $(r, l)=1$ and $(s, k)=1$. Now the statement of this lemma follows from Lemma $3.3 \mathrm{c})$. 
e) We know from Lemma $3.3 \mathrm{c}$ ) that we only have to consider characters $\chi_{i}, 1 \leq i \leq 3$, that are primitive modulo $k^{2}$. We know from $[3$, Lemma $5.1 \mathrm{c}]$, that for a primitive character $\chi_{i}$ modulo $k^{2}$, we have $\chi_{i}(1+\bar{b} s k)=e\left(c_{i} \bar{b} s / k\right)$, where $\left(k, c_{i}\right)=1$ and $\bar{b} b \equiv 1\left(\bmod k^{2}\right)$. By definition,

$$
\begin{aligned}
C\left(\chi_{i}, k^{2}, k, b_{i}, a\right) & =\sum_{s=1}^{k} \chi_{i}\left(b_{i}+s k\right) e\left(\frac{a b_{i}+a k s}{k^{2}}\right) \\
& =\chi_{i}\left(b_{i}\right) \sum_{s=1}^{k} \chi_{i}\left(1+\bar{b}_{i} s k\right) e\left(\frac{a b_{i}+a k s}{k^{2}}\right) \\
& =\chi_{i}\left(b_{i}\right) \sum_{s=1}^{k} e\left(\frac{s c_{i} \bar{b}_{i}}{k^{2}}\right) e\left(\frac{a b_{i}+a k s}{k^{2}}\right) .
\end{aligned}
$$

Inserting (3.1) in the definition of $Z(\ldots)$, we find

$$
\begin{aligned}
= & \frac{\prod_{i=1}^{3} \chi_{i}\left(b_{i}\right)}{\phi^{3}\left(k^{2}\right)} \sum_{a=1}^{k^{2}} \prod_{i=1}^{3}\left(\sum_{s_{i}=1}^{k} e\left(\frac{s_{i} c_{i} \bar{b}_{i}}{k^{2}}\right) e\left(\frac{a b_{i}+a k s_{i}}{k^{2}}\right)\right) e\left(\frac{-a N}{k^{2}}\right) \\
= & \frac{\prod_{i=1}^{3} \chi_{i}\left(b_{i}\right)}{\phi^{3}\left(k^{2}\right)} \sum_{s_{1}}^{k} \sum_{s_{2}}^{k} \sum_{s_{3}}^{k} e\left(\frac{s_{1} c_{1} \bar{b}_{1}+s_{2} c_{2} \bar{b}_{2}+s_{3} c_{3} \bar{b}_{3}}{k^{2}}\right) \\
& \times \sum_{a=1}^{k^{2}} e\left(\frac{a\left(b_{1}+b_{2}+b_{3}-N+s_{1} k+s_{2} k+s_{3} k\right)}{k^{2}}\right) .
\end{aligned}
$$

Using that $b_{1}+b_{2}+b_{3}-N=M k, M \in \mathbf{Z}$, we can write the inner sum in (3.2) as:

$$
\begin{aligned}
\sum_{a=1}^{k^{2}} *\left(\frac{a k\left(M+s_{1}+s_{2}+s_{3}\right)}{k^{2}}\right) & \\
& =k \sum_{a=1}^{k-1} e\left(\frac{a\left(M+s_{1}+s_{2}+s_{3}\right)}{k}\right) \\
& = \begin{cases}k(k-1) & \text { if } M+s_{1}+s_{2}+s_{3} \equiv 0(\bmod k), \\
-k & \text { else. }\end{cases}
\end{aligned}
$$


Obviously,

$$
\sharp\left\{s_{1}, s_{2}, s_{3}: 1 \leq s_{1}, s_{2}, s_{3} \leq k, M+s_{1}+s_{2}+s_{3} \equiv 0(\bmod k)\right\}=k^{2} .
$$

Thus, noting that $k / \phi(k) \leq 2$, we obtain from (3.2) and (3.3):

$$
Z\left(N, k^{2}, k, \chi_{1}, \chi_{2}, \chi_{3}\right) \ll k^{-6} k^{4}=k^{-2} \text {. }
$$

Lemma 3.4. Let there be given primitive characters $\chi_{i} \bmod r_{i}$, $i=1,2,3$, the principal character $\chi_{0} \bmod q$ and $r=\left[r_{1}, r_{2}, r_{3}\right]$.

a) If $(r, k)=1$, then

$$
\sum_{\substack{q \leq P \\ r \mid q}}\left|Z\left(N, q, k_{q}, \chi_{1} \chi_{0}, \chi_{2} \chi_{0}, \chi_{3} \chi_{0}\right)\right| \ll r^{-1 / 2} L .
$$

b) If $k^{m} \| r, m \in\{1,2\}$, then

$$
\sum_{\substack{q \leq P \\ r \mid q}}\left|Z\left(N, q, k_{q}, \chi_{1} \chi_{0}, \chi_{2} \chi_{0}, \chi_{3} \chi_{0}\right)\right| \ll s^{-1 / 2} k^{-2} L .
$$

c) If $(r, k)=1$, then

$$
\sum_{\substack{q \leq P \\ k r \mid q}}\left|Z\left(N, q, k_{q}, \chi_{1} \chi_{0}, \chi_{2} \chi_{0}, \chi_{3} \chi_{0}\right)\right| \ll r^{-1 / 2} k^{-2} L .
$$

Proof. a) Let $J$ denote the left-hand side in Lemma 3.4 a). Arguing as in the proof of Lemma $3.3 \mathrm{~d}$ ), we see that we can focus on terms $Z(N, q, \ldots)$ which can be written as follows

$$
Z\left(N, q, k_{q}, \ldots\right)=Z(N, r, 1, \ldots) A\left(N, l, k_{l}\right)
$$

where $(l, r)=1$. Thus

$$
J \ll|Z(N, r, 1, \ldots)| \sum_{l \leq P / r}\left|A\left(N, l, k_{l}\right)\right| .
$$


From Lemma 3.3 a), we derive

$$
|Z(N, r, 1, \ldots)| \ll r^{-3} r r^{3 / 2} L_{2}^{3}=r^{-1 / 2} L_{2}^{3} .
$$

Lemma $3.3 \mathrm{~b})$ implies that $\left|A\left(N, l, k_{l}\right)\right| \leq l \phi^{-3}(l)$. Thus

$$
\sum_{l \leq P / r} A\left(N, l, k_{l}\right) \ll 1 .
$$

Part a) follows from (3.4)-(3.6). For the proof of part b), we use the definition (2.3) and Lemma $3.2 \mathrm{~b}$ ) to write

$$
Z(N, r, k, \ldots)=Z(N, s, 1, \ldots) Z\left(N, k^{m}, k, \ldots\right) .
$$

As in $(3.5)$, we use Lemma 3.3 a) to estimate $Z(N, s, 1, \ldots)$. In order to estimate $Z\left(N, k^{m}, k, \ldots\right)$, for $m=1$, we use the fact that by definition $|C(\chi, k, k, b, a)| \leq 1$ whereas for $m=2$ we use Lemma $3.3 \mathrm{e})$. Thus,

$$
Z(N, s, 1, \ldots) Z\left(N, k^{m}, k, \ldots\right) \ll s^{-1 / 2} k^{-2} L_{2}^{3} .
$$

The lemma then follows from (3.4), (3.6), (3.7) and (3.8). For the proof of part c), we argue as in (3.4):

$$
J \ll|Z(N, r, 1, \ldots)| \sum_{\substack{l \leq P / r \\ k \mid l}}|A(N, l, k)| .
$$

We see from Lemma $3.3 \mathrm{~b}$ ) that

$$
\sum_{\substack{l \leq P / r \\ k \mid l}}|A(N, l, k)| \leq \sum_{\substack{l \leq P / r \\ k \mid l}} \frac{l}{l^{3}} L_{2}^{3} \leq k^{-2} L_{2}^{3} \sum_{l \leq P / r k} l^{-2} \ll k^{-2} L_{2}^{3}
$$

Part c) now follows from (3.5), (3.9) and (3.10).

Lemma 3.5. There exists a positive number $J$ such that:

a)

$$
\sum_{q=1}^{\infty} \frac{1}{\phi\left(k / k_{q}\right)^{3}} A\left(N, q, k_{q}\right)=\sigma(N, k) .
$$


b)

$$
\sum_{q \geq P} \frac{1}{\phi\left(k / k_{q}\right)^{3}}\left|A\left(N, q, k_{q}\right)\right| \ll(P k)^{-1} L^{J}
$$

Proof. The proof is nearly identical to the proof of Lemma 4.6 in [2]. Whereas in $[\mathbf{2}]$ the estimate $k / \phi(k) \ll k^{\varepsilon}$ is used, here the estimate $k / \phi(k) \ll \log \log k$ is applied.

4. Treatment of the major arcs. We first consider the set $E_{1}(k)$. If $k \nmid q$, we find

$$
S\left(\frac{a}{q}+\lambda, b_{i}\right)=\sum_{g=1}^{q} *^{*} e\left(\frac{g a}{q}\right) \sum_{\substack{N / 4<n \leq N \\ n \equiv b_{i}(\bmod k) \\ n \equiv g(\bmod q)}} \Lambda(n) e(n \lambda)+O\left(L^{2}\right) .
$$

We shall introduce the Dirichlet characters $\xi \bmod k$ and $\chi \bmod q$ and obtain

$$
\begin{aligned}
S\left(\frac{a}{q}+\lambda, b_{i}\right)= & \frac{1}{\phi(k) \phi(q)} C\left(\chi_{0}, q, 1, b_{i}, a\right) T(\lambda)+\frac{1}{\phi(k) \phi(q)} \\
& +\sum_{\xi \bmod k} \bar{\xi}\left(b_{i}\right) \sum_{\chi \bmod q} C\left(\bar{\chi}, q, 1, b_{i}, a\right) W(\lambda, \xi \chi)+O\left(L^{2}\right) .
\end{aligned}
$$

In the sequel, we will neglect the error term $O\left(L^{2}\right)$. We will see that its contribution will be dominated by other, larger error terms. We obtain from (2.5):

$$
R_{1}(N)=R_{1}^{m}(N)+R_{1}^{e}(N),
$$

where

$$
\begin{aligned}
R_{1}^{m}(N)= & \sum_{\substack{q \leq P_{1} \\
k \nmid q}} \frac{1}{\phi^{3}(k) \phi^{3}(q)} \sum_{a=1}^{q} \prod_{i=1}^{3} C\left(\chi_{0}, q, 1, b_{i}, a\right) e\left(-\frac{a}{q} N\right) \\
& \times \int_{-1 / q Q}^{1 / q Q} T^{3}(\lambda) e(-N \lambda) d \lambda
\end{aligned}
$$




$$
\begin{aligned}
R_{1}^{e}(N)= & \sum_{\substack{q \leq P_{1} \\
k \nmid q}} \frac{1}{\phi^{3}(k) \phi^{3}(q)} \sum_{a=1}^{q}{ }^{*} e\left(-\frac{a}{q} N\right) \\
& \times \int_{-1 / q Q}^{1 / q Q} \prod_{i=1}^{3}\left(\sum_{\xi \bmod k} \bar{\xi}\left(b_{i}\right) \sum_{\chi \bmod q} C\left(\bar{\chi}, q, 1, b_{i}, a\right) W(\lambda, \xi \chi)\right) \\
& \times e(-\lambda N) d \lambda \\
& +\sum_{i=1}^{3} \sum_{\substack{q \leq P_{1} \\
k \nmid q}} \frac{1}{\phi^{3}(k) \phi^{3}(q)} \sum_{a=1}^{q} e\left(-\frac{a}{q} N\right) \\
& \times \int_{-1 / q Q}^{1 / q Q} \prod_{j=1}^{3}\left(\sum_{\xi \bmod k} \bar{\xi}\left(b_{j}\right) \sum_{\chi \bmod q} C\left(\bar{\chi}, q, 1, b_{j}, a\right) W(\lambda, \xi \chi)\right) \\
& \times C\left(\chi_{0}, q, 1, b_{i}, a\right) T(\lambda) e(-\lambda N) d \lambda \\
& +\sum_{i=1}^{3} \sum_{q \leq P_{1}} \frac{1}{\phi^{3}(k) \phi^{3}(q)} \sum_{a=1}^{*} e\left(-\frac{a}{q} N\right) \\
& \times \int_{-1 / q Q}^{1 / q Q}\left(\sum_{\xi \bmod k} \bar{\xi}\left(b_{i}\right) \sum_{\chi \bmod q} C\left(\bar{\chi}, q, 1, b_{i}, a\right) W(\lambda, \xi \chi)\right) \\
& \times \prod_{j=1}^{3} C\left(\chi_{0}, q, 1, b_{j}, a\right) T^{2}(\lambda) e(-\lambda N) d \lambda \\
= & \sum_{1}+\sum_{j}+\sum_{3} .
\end{aligned}
$$

We first evaluate the main term $R_{1}^{m}(N)$ using (3.6) with $r=1$,

$$
\begin{aligned}
R_{1}^{m}(N)= & \frac{1}{\phi^{3}(k)} \sum_{\substack{q \leq P_{1} \\
k \nmid q}} A(N, q, 1) \int_{-1 / 2}^{1 / 2} T(\lambda)^{3} e(-N \lambda) d \lambda \\
& +O\left(\frac{1}{\phi^{3}(k)} \sum_{\substack{q \leq P_{1} \\
k \nmid q}}|A(N, q, 1)| \int_{1 / q Q}^{1 / 2} \frac{1}{|\lambda|^{3}} d \lambda\right)
\end{aligned}
$$




$$
\begin{aligned}
& =\frac{1}{\phi^{3}(k)} \sum_{\substack{q \leq P_{1} \\
k \nmid q}} A(N, q, 1) \frac{N^{2}}{32}+O\left(\frac{\left(P_{1} Q\right)^{2}}{\phi^{3}(k)}\right) \\
& =\frac{1}{\phi^{3}(k)} \sum_{\substack{q \leq P_{1} \\
k \nmid q}} A(N, q, 1) \frac{N^{2}}{32}+O\left(N^{2} k^{-4} L^{-A}\right),
\end{aligned}
$$

where we have used $T(\lambda) \ll 1 /|\lambda|$ and

$$
\int_{-1 / 2}^{1 / 2} T(\lambda)^{3} e(-N \lambda) d \lambda=\sum_{N / 4<n_{1}<N / 2} \sum_{N / 4<n_{2}<3 N / 4-n_{1}} 1=\frac{N^{2}}{32}+O(N) .
$$

In the sequel we will without further mention use the fact that, for any character $\chi$ induced by a primitive character $\chi^{*}$, we have $W(\chi, \chi \xi)=$ $W\left(\lambda, \chi^{*} \xi\right)+O\left(L^{2}\right)$. Using Lemma 3.4 a), we estimate $\sum_{1}$ :

$$
\begin{aligned}
& \left|\sum_{1}\right| \leq \frac{1}{\phi^{3}(k)} \sum_{\substack{q \leq P_{1} \\
k \nmid q}} \sum_{\chi_{1} \bmod q} \sum_{\chi_{2} \bmod q} \sum_{\chi_{3} \bmod q} \sum_{\xi_{1} \bmod k} \sum_{\xi_{2} \bmod k} \sum_{\xi_{3} \bmod k} \\
& \times\left|Z\left(N, q, 1, \chi_{1}, \chi_{2}, \chi_{3}\right)\right| \int_{-1 / q Q}^{1 / q Q} \prod_{j=1}^{3}\left|W\left(\lambda, \chi_{j} \xi_{j}\right)\right| d \lambda \\
& \leq \frac{1}{\phi^{3}(k)} \sum_{\substack{r_{1} \leq P_{1} \\
k \nmid r_{1}}} \sum_{\substack{r_{2} \leq P_{1} \\
k \nmid r_{2}}} \sum_{\substack{r_{3} \leq P_{1} \\
k \nmid r_{3}}} \sum_{\chi_{1} \bmod r_{1}} * \sum_{\chi_{2} \bmod r_{2}} * \sum_{\chi_{3} \bmod r_{3}}^{*} \\
& \times \sum_{\xi_{1} \bmod k} \sum_{\xi_{2} \bmod k} \sum_{\xi_{3} \bmod k} \\
& \times \int_{-1 /\left[r_{1}, r_{2}, r_{3}\right] Q}^{1 /\left[r_{1}, r_{2}, r_{3}\right] Q} \prod_{j=1}^{3}\left(\left|W\left(\lambda, \chi_{j} \xi_{j}\right)\right|+L^{2}\right) d \lambda \\
& \times \sum_{\substack{q \leq P_{1} \\
\left[r_{1}, r_{2}, r_{3}\right] \mid q}}\left|Z\left(N, q, 1, \chi_{1} \chi_{0}, \chi_{2} \chi_{0}, \chi_{3} \chi_{0}\right)\right| \\
& \ll \frac{L}{\phi^{3}(k)} \sum_{\substack{r_{1} \leq P_{1} \\
k \nmid r_{1}}} \sum_{\substack{r_{2} \leq P_{1} \\
k \nmid r_{2}}} \sum_{\substack{r_{3} \leq P_{1} \\
k \nmid r_{3}}}\left[r_{1}, r_{2}, r_{3}\right]^{-1 / 2}
\end{aligned}
$$




$$
\begin{aligned}
& \times \sum_{\chi_{1} \bmod r_{1}}^{*} \sum_{\chi_{2} \bmod r_{2}}^{*} \sum_{\chi_{3} \bmod r_{3}} * \sum_{\xi_{1} \bmod k} \sum_{\xi_{2} \bmod k} \sum_{\xi_{3} \bmod k} \\
& \times \int_{-1 /\left[r_{1}, r_{2}, r_{3}\right] Q}^{1 /\left[r_{1}, r_{2}, r_{3}\right] Q} \prod_{j=1}^{3}\left(\left|W\left(\lambda, \chi_{j} \xi_{j}\right)\right|+L^{2}\right) d \lambda,
\end{aligned}
$$

In the following, we will neglect the error terms $L^{2}$ in the last integral in (4.5) as their contribution will be dominated by other terms. As a character $\xi$ modulo $k$ is either primitive or the principal character modulo $k$, the following relation holds for all characters $\chi_{i}$ and $\xi_{i}$, $i \in\{1,2,3\}$, over which is summed in (4.5):

$$
(\chi \xi)^{*}= \begin{cases}\chi^{*} & \text { if } \xi=\xi_{0} \\ \chi^{*} \xi & \text { otherwise }\end{cases}
$$

Thus we see from (4.5) and (4.6) and by the notation for $s_{i}$ introduced in $(2.2)$,

$$
\begin{aligned}
\sum_{1} \ll & k^{-3} L^{2}\left(\sum_{\substack{r_{1} \leq P_{1} \\
k \| \mid r_{1}}} \sum_{\substack{r_{2} \leq P_{1} \\
k \| \mid r_{2}}} \sum_{\substack{r_{3} \leq P_{1} k \\
k \| r_{3}}}+\sum_{\substack{r_{1} \leq P_{1} k\left\|r_{1} \\
k\right\| r_{2} \leq P_{1} k \| r_{2}}} \sum_{\substack{r_{3} \leq P_{1} \\
k \nmid r_{3}}} \sum+\sum_{\substack{r_{1} \leq P_{1} \\
k \| r_{1}}} \sum_{\substack{r_{2} \leq P_{1} \\
k \nmid r_{2}}}+\sum_{\substack{r_{3} \leq P_{1} \\
k \nmid r_{3}}} \sum_{\substack{r_{1} \leq P_{1} \\
k \nmid r_{1}}} \sum_{\substack{r_{2} \leq P_{1} \\
k \nmid r_{2}}}\right)\left[s_{1}, s_{2}, s_{3}\right]^{-1 / 2} r_{1} \\
& \times \sum_{\chi_{1} \bmod r_{1}} * \sum_{\chi_{2} \bmod r_{2}} * \sum_{\chi_{3} \bmod r_{3}} * \int_{-1 /\left[s_{1}, s_{2}, s_{3}\right] Q}^{1 /\left[s_{1}, s_{2}, s_{3}\right] Q} \prod_{j=1}^{3}\left|W\left(\lambda, \chi_{j}\right)\right| d \lambda \\
= & : \sum_{i=1}^{4} \sum_{1, i},
\end{aligned}
$$

where each $\sum_{1, i}$ stands for one of the multiple sums in (4.7). Using 
$\left[s_{1}, s_{2}, s_{3}\right]^{1 / 2} \geq s_{2}^{1 / 4} s_{3}^{1 / 4}$, we obtain

$$
\begin{aligned}
\sum_{1,1} \ll & k^{-3} L^{2} \sum_{\substack{r_{1} \leq P_{1} k \\
k \mid r_{1}}} \sum_{\chi_{1} \bmod r_{1}}{ }^{*} \max _{|\lambda| \leq 1 / s_{1} Q}\left|W\left(\lambda, \chi_{1}\right)\right| \\
& \times \sum_{\substack{r_{2} \leq P_{1} k \\
k \mid r_{2}}} s_{2}^{-1 / 4} \sum_{\chi_{2} \bmod r_{2}} *\left(\int_{-1 / s_{2} Q}^{1 / s_{2} Q}\left|W\left(\lambda, \chi_{2}\right)\right|^{2} d \lambda\right)^{1 / 2} \\
& +\sum_{\substack{r_{3} \leq P_{1} k \\
k \mid r_{3}}} s_{3}^{-1 / 4} \sum_{\chi_{3} \bmod r_{3}} *\left(\int_{-1 / s_{3} Q}^{1 / s_{3} Q}\left|W\left(\lambda, \chi_{3}\right)\right|^{2} d \lambda\right)^{1 / 2} \\
= & : k^{-2} L^{2} I_{A} W_{A}^{2},
\end{aligned}
$$

where

$$
\begin{aligned}
I_{A} & =k^{-1 / 3} \sum_{\substack{r \leq P_{1} k \\
k \mid r}} \sum_{(\bmod r)}^{*} \max _{|\lambda| \leq k / r Q}|W(\lambda, \chi)|, \\
W_{A} & =k^{-1 / 3} \sum_{\substack{r \leq P_{1} k \\
k \mid r}}\left(\frac{r}{k}\right)^{-1 / 4} \sum_{\chi(\bmod r)} *\left(\int_{-k / r Q}^{k / r Q}|W(\lambda, \chi)|^{2} d \lambda\right)^{1 / 2} .
\end{aligned}
$$

Arguing similarly, we obtain

$$
\sum_{i=2}^{4} \sum_{1, i} \ll k^{-2} L^{2}\left(I_{A} W_{A} W_{B}+I_{A} W_{B}^{2}+I_{B} W_{B}^{2}\right)
$$

where

$$
\begin{aligned}
I_{B} & =k^{-1 / 3} \sum_{\substack{r \leq P_{1} \\
k \nmid r}} \sum_{(\bmod r)}{ }^{*} \max _{|\lambda| \leq 1 / r Q}|W(\lambda, \chi)|, \\
W_{B} & =k^{-1 / 3} \sum_{\substack{r \leq P_{1} \\
k \nmid r}} r^{-1 / 4} \sum_{\chi(\bmod r)} *\left(\int_{-1 / r Q}^{1 / r Q}|W(\lambda, \chi)|^{2} d l\right)^{1 / 2} .
\end{aligned}
$$


In the same way we find

$$
\begin{aligned}
\sum_{2}+\sum_{3} \ll & k^{-2} L^{2} \max _{|\lambda| \leq 1 / Q}|T(\lambda)|\left(W_{B}^{2}+W_{B} W_{A}+W_{A}^{2}\right) \\
& +k^{-2} L^{2} \max _{|\lambda| \leq 1 / Q}|T(\lambda)|\left(\int_{-1 / Q}^{1 / Q}|T(\lambda)|^{2} d l\right)^{1 / 2}\left(W_{B}+W_{A}\right) .
\end{aligned}
$$

We have

$$
\max _{|\lambda| \leq 1 / Q}|T(\lambda)| \ll N
$$

Using $T(\lambda) \leq \min (N,(1 / \lambda))$, we see that

$$
\left(\int_{-1 / Q}^{1 / Q}|T(\lambda)|^{2} d l\right)^{1 / 2} \ll N^{1 / 2} .
$$

Therefore, we see from (4.2) and (4.7)-(4.12):

$$
\begin{aligned}
R_{1}^{e}(N) \ll & k^{-2} L^{2}\left(N\left(W_{B}^{2}+W_{B} W_{A}+W_{A}^{2}\right)+N^{3 / 2}\left(W_{B}+W_{A}\right)\right. \\
& \left.+I_{A} W_{A}^{2}+I_{A} W_{A} W_{B}+I_{A} W_{B}^{2}+I_{B} W_{B}^{2}\right) .
\end{aligned}
$$

For $q \in E_{2}(k)$, we see

$$
\begin{aligned}
S\left(\frac{a}{q}+\lambda, b_{i}\right)= & \sum_{\substack{g=1 \\
g \equiv b_{i}(\bmod k)}}^{q} e\left(\frac{g a}{q}\right) \sum_{\substack{N / 4<n \leq N \\
n \equiv b_{i}(\bmod k) \\
n \equiv g(\bmod q)}} \Lambda(n) e(n \lambda) \\
= & \sum_{\substack{g=1 \\
g \equiv b_{i}(\bmod k)}}^{q} e\left(\frac{g a}{q}\right) \sum_{\substack{N / 4<n \leq N \\
n \equiv g(\bmod q)}} \Lambda(n) e(n \lambda) \\
= & \frac{1}{\phi(q)} C\left(\chi_{0}, q, k, b_{i}, a\right) T(\lambda) \\
& +\frac{1}{\phi(q)} \sum_{\chi \bmod q} C\left(\bar{\chi}, q, k, b_{i}, a\right) W(\lambda, \chi) .
\end{aligned}
$$


Arguing as in (4.1)-(4.3), we obtain by applying (3.6) in the same way as in (4.3) and using (4.4):

$$
R_{2}(N)=R_{2}^{m}(N)+R_{2}^{e}(N)
$$

where

$$
R_{2}^{m}(N)=\sum_{\substack{q \leq P_{2} \\ k \mid q}} A(N, q, k) \frac{N^{2}}{32}+O\left(N^{2} k^{-3} L^{-A}\right)
$$

$$
\begin{aligned}
& R_{2}^{e}(N)=\sum_{\substack{q \leq P_{2} \\
k \mid q}} \frac{1}{\phi^{3}(q)} \sum_{a=1}^{q} * \int_{-1 / q Q}^{1 / q Q} \\
& \times \prod_{i=1}^{3}\left(\sum_{\chi \bmod q} C\left(\bar{\chi}, q, k, b_{i}, a\right) W(\lambda, \chi)\right) e\left(-\frac{a}{q} N-\lambda N\right) d \lambda \\
& +\sum_{i=1}^{3} \sum_{\substack{q \leq P_{2} \\
k \mid q}} \frac{1}{\phi^{3}(q)} \sum_{a=1}^{q} * \int_{-1 / q Q}^{1 / q Q} \\
& \times \prod_{\substack{j=1 \\
j \neq i}}^{3}\left(\sum_{\chi \bmod q} C\left(\bar{\chi}, q, k, b_{j}, a\right) W(\lambda, \chi)\right) \\
& \times C\left(\chi_{0}, q, k, b_{i}, a\right) T(\lambda) e\left(-\frac{a}{q} N-\lambda N\right) d \lambda \\
& +\sum_{i=1}^{3} \sum_{\substack{q \leq P_{2} \\
k \mid q}} \frac{1}{\phi^{3}(q)} \sum_{a=1}^{q} \int_{-1 / q Q}^{1 / q Q} \sum_{\chi \bmod q} C\left(\bar{\chi}, q, k, b_{i}, a\right) W(\lambda, \chi) \\
& \times \prod_{\substack{j=1 \\
j \neq i}}^{3} C\left(\chi_{0}, q, k, b_{j}, a\right) T^{2}(\lambda) e\left(-\frac{a}{q} N-\lambda N\right) d \lambda \\
& =: \sum_{4}+\sum_{5}+\sum_{6} \text {. }
\end{aligned}
$$


Arguing similarly as in (4.5) and using Lemma $3.3 \mathrm{~d}$ ), we see

$$
\begin{aligned}
\sum_{4}= & \sum_{\substack{q \leq P_{2} \\
k \mid q}} \sum_{\chi_{1} \bmod q} \sum_{\chi_{2} \bmod q} \sum_{\chi_{3} \bmod q}\left|Z\left(N, q, k, \chi_{1}, \chi_{2}, \chi_{3}\right)\right| \\
& \times \int_{-1 / q Q}^{1 / q Q} \prod_{j=1}^{3}\left|W\left(\lambda, \chi_{j}\right)\right| d \lambda
\end{aligned}
$$

$$
\begin{aligned}
& \ll\left(\sum_{\substack{r_{1} \leq P_{2} \\
k \| r_{1}}} \sum_{\substack{r_{2} \leq P_{2} \\
k \| \mid r_{2}}} \sum_{\substack{r_{3} \leq P_{2} \\
k \| r_{3}}}+\sum_{\substack{r_{1} \leq P_{2} \\
k \| r_{1}}} \sum_{\substack{r_{2} \leq P_{2} \\
k \| r_{2}}} \sum_{\substack{r_{3} \leq P_{2} / k \\
k \nmid r_{3}}}\right. \\
& +\sum_{\substack{r_{1} \leq P_{2} \\
k \| r_{1}}} \sum_{\substack{r_{2} \leq P_{2} / k \\
k \nmid r_{2}}} \sum_{\substack{r_{3} \leq P_{2} / k \\
k \nmid r_{3}}}+\sum_{\substack{r_{1} \leq P_{2} / k \\
k \nmid r_{1}}} \sum_{\substack{r_{2} \leq P_{2} / k \\
k \nmid r_{2}}} \sum_{\substack{r_{3} \leq P_{2} / k \\
k \nmid r_{3}}} \\
& +\sum_{\substack{r_{1} \leq P_{2} \\
k^{2} \| r_{1}}} \sum_{\substack{r_{2} \leq P_{2} \\
k^{2} \| r_{2}}} \sum_{\substack{r_{3} \leq P_{2} \\
k^{2} \| r_{3}}} \sum_{\chi_{1} \bmod r_{1}} * \sum_{\chi_{2} \bmod r_{2}} * \sum_{\chi_{3} \bmod r_{3}} * \\
& \times \int_{-1 /\left[r_{1}, r_{2}, r_{3}\right] Q}^{1 /\left[r_{1}, r_{2}, r_{3}\right] Q} \prod_{j=1}^{3}\left(\left|W\left(\lambda, \chi_{j}\right)\right|+L^{2}\right) d \lambda \\
& \times \sum_{\substack{q \leq P_{2} \\
\left[r_{1}, r_{2}, r_{3}\right]|q \\
k| q}}\left|Z\left(N, q, k_{q}, \chi_{1} \chi_{0}, \chi_{2} \chi_{0}, \chi_{3} \chi_{0}\right)\right| \\
& =: \sum_{i=1}^{5} \sum_{4, i}
\end{aligned}
$$

where each $\sum_{4, i}$ stands for one of the multiple sums in (4.17). The condition $k \mid q$ in the index of the sum $\sum_{\substack{q \leq P_{2} \\\left[r_{1}, r_{2}, r_{3}\right] \mid q}} \mid Z\left(N, q, k_{q}, \chi_{1} \chi_{0}\right.$, $\left.\chi_{2} \chi_{0}, \chi_{3} \chi_{0}\right)$ is only necessary for the sum $\sum_{4,4}^{k \mid q}$. In the other cases, $k \mid\left[r_{1}, r_{2}, r_{3}\right]$ which implies $k \mid q$. Thus, we will only make use of the condition when we estimate the sum $\sum_{4,4}$. Again, we neglect the error terms $L^{2}$ in the last expression as they will be dominated in the sequel by other error terms. In order to estimate the $\sum_{4,1}$, we use the fact that for all $q$ considered in (4.17), there holds $k^{3} \nmid q$ because of $q \leq P_{2}$. 
This allows us to apply Lemma $3.4 \mathrm{~b}$ ). Using (2.2), Lemma $3.4 \mathrm{~b}$ ) and the relation $\left[s_{1}, s_{2}, s_{3}\right]^{1 / 2} \geq s_{1}^{1 / 6} s_{2}^{1 / 6} s_{3}^{1 / 6}$, we argue as in (4.8):

$$
\begin{aligned}
\sum_{4,1} & \ll k^{-2} L \sum_{\substack{r_{1} \leq P_{2} \\
k \| r_{1}}} \sum_{\substack{r_{2} \leq P_{2} \\
k \| r_{2}}} \sum_{\substack{r_{3} \leq P_{2} \\
k \| r_{3}}}\left[s_{1}, s_{2}, s_{3}\right]^{-1 / 2} \\
& +\sum_{\chi_{1} \bmod r_{1}}^{*} \sum_{\chi_{2} \bmod r_{2}} \sum_{\chi_{3} \bmod r_{3}}^{*} \int_{-1 /\left[r_{1}, r_{2}, r_{3}\right] Q}^{*} \prod_{j=1}^{1 /\left[r_{1}, r_{2}, r_{3}\right] Q}\left|W\left(\lambda, \chi_{j}\right)\right| d \lambda \\
\ll & \ll k^{-2} L I_{C} W_{C}^{2},
\end{aligned}
$$

where

$$
\begin{aligned}
I_{C} & =\sum_{\substack{r \leq P_{2} \\
k \mid r}}\left(\frac{r}{k}\right)^{-1 / 6} \sum_{\chi(\bmod r)} * \max _{|\lambda| \leq 1 / r Q}|W(\lambda, \chi)|, \\
W_{C} & =\sum_{\substack{r \leq P_{2} \\
k \mid r}}\left(\frac{r}{k}\right)^{-1 / 6} \sum_{\chi(\bmod r)} *\left(\int_{-1 / r Q}^{1 / r Q}|W(\lambda, \chi)|^{2} d \lambda\right)^{1 / 2} .
\end{aligned}
$$

Arguing as in (4.10), using Lemma $3.4 \mathrm{~b}$ ) and the relation $\left[s_{1}, s_{2}, s_{3}\right]^{1 / 2}$ $\geq s_{1}^{1 / 6} s_{2}^{1 / 6} s_{3}^{1 / 6}$, we obtain

$$
\sum_{4,2}+\sum_{4,3} \leq k^{-2} L\left(I_{D} W_{C}^{2}+I_{D} W_{D} W_{C}\right)
$$

where

$$
\begin{aligned}
I_{D} & =\sum_{\substack{r \leq P_{2} / k \\
k \nmid r}} \sum_{(\bmod r)} * \max _{|\lambda| \leq 1 / r Q}|W(\lambda, \chi)|, \\
W_{D} & =\sum_{\substack{r \leq P_{2} / k \\
k \nmid r}} r^{-1 / 4} \sum_{(\bmod r)} *\left(\int_{-1 / r Q}^{1 / r Q}|W(\lambda, \chi)|^{2} d \lambda\right)^{1 / 2} .
\end{aligned}
$$


For the estimate of $\sum_{4,4}$, we argue as in (4.19) and apply Lemma $3.4 \mathrm{c}$ ):

$$
\begin{aligned}
\sum_{4,4} \ll & k^{-2} L \sum_{\substack{r_{1} \leq P_{2} / k \\
k \nmid r_{1}}} \sum_{\substack{r_{2} \leq P_{2} / k \\
k \nmid r_{2}}} \sum_{\substack{r_{3} \leq P_{2} / k \\
k \nmid r_{3}}}\left[r_{1}, r_{2}, r_{3}\right]^{-1 / 2} \\
& +\sum_{\chi_{1} \bmod r_{1}} * \sum_{\chi_{2} \bmod r_{2}}^{*} \sum_{\chi_{3} \bmod r_{3}} * \int_{-1 /\left[r_{1}, r_{2}, r_{3}\right] Q}^{1 /\left[r_{1}, r_{2}, r_{3}\right] Q} \prod_{j=1}^{3}\left|W\left(\lambda, \chi_{j}\right)\right| d \lambda \\
\leq & k^{-2} L I_{D} W_{D}^{2} .
\end{aligned}
$$

As $k^{3} \nmid q$ for all considered $q$, we use Lemma 3.4 b) to estimate the sum $\sum_{4,5}$ :

$$
\begin{aligned}
\sum_{4,5} \ll & k^{-2} L \sum_{\substack{r_{1} \leq P_{2} \\
k^{2} \| r_{1}}} \sum_{\substack{r_{2} \leq P_{2} \\
k^{2} \| r_{2}}} \sum_{r_{3} \leq P_{2}{ }^{2} \| r_{3}}\left[s_{1}, s_{2}, s_{3}\right]^{-1 / 2} \\
& +\sum_{\chi_{1} \bmod r_{1}} * \sum_{\chi_{2} \bmod r_{2}} * \sum_{\chi_{3} \bmod r_{3}}{ }^{*} \int_{-1 /\left[r_{1}, r_{2}, r_{3}\right] Q}^{1 /\left[r_{1}, r_{2}, r_{3}\right] Q} \prod_{j=1}^{3}\left|W\left(\lambda, \chi_{j}\right)\right| d \lambda \\
\leq & k^{-2} L I_{E} W_{E}^{2},
\end{aligned}
$$

where

$$
\begin{aligned}
I_{E} & =\sum_{\substack{r \leq P_{2} \\
k^{2} \mid r}}\left(\frac{r}{k^{2}}\right)^{-1 / 6} \sum_{\chi(\bmod r)} * \max _{|\lambda| \leq 1 / r Q}|W(\lambda, \chi)| \\
W_{E} & =\sum_{\substack{r \leq P_{2} \\
k^{2} \mid r}}\left(\frac{r}{k^{2}}\right)^{-1 / 6} \sum_{\substack{\bmod r)}} *\left(\int_{-1 / r Q}^{1 / r Q}|W(\lambda, \chi)|^{2} d \lambda\right)^{1 / 2} .
\end{aligned}
$$

Arguing as in (4.9), we obtain

$$
\begin{aligned}
\sum_{5}+\sum_{6} \ll & k^{-2} L\left(\max _{|l| \leq 1 / Q}|T(\lambda)|\left(W_{C}^{2}+W_{C} W_{D}+W_{D}^{2}+W_{E}^{2}\right)\right. \\
& \left.+\max _{|\lambda| \leq 1 / Q}|T(\lambda)|\left(\int_{-1 / Q}^{1 / Q}|T(\lambda)|^{2} d l\right)^{1 / 2}\left(W_{C}+W_{D}+W_{E}\right)\right) .
\end{aligned}
$$


Therefore, we see from $(4.11),(4.12)$, and (4.16)-(4.23):

$$
\begin{aligned}
R_{2}^{e}(N) \ll & k^{-2} L\left(N\left(W_{C}^{2}+W_{D} W_{C}+W_{D}^{2}+W_{E}^{2}\right)\right. \\
& +N^{3 / 2}\left(W_{C}+W_{D}+W_{E}\right) \\
& \left.+I_{C} W_{C}^{2}+I_{D} W_{C}^{2}+I_{D} W_{D} W_{C}+I_{D} W_{D}^{2}+I_{E} W_{E}^{2}\right) .
\end{aligned}
$$

Using Lemma 3.5, we see from (4.3) and (4.15) that for a sufficiently large $G=G(A)$

$$
R_{1}^{m}(N)+R_{2}^{m}(N)=\sigma(N, k) \frac{N^{2}}{32}+O\left(N^{2} k^{-2} L^{-A}\right) .
$$

Thus we see from $(4.1),(4.13),(4.14),(4.24)$ and (4.25) that the proof of (2.7) reduces to the proof of the following two lemmas:

Lemma 4.1. If $k \leq N^{(2 / 15)-\varepsilon}$, then for $F \in\{A, B, D\}$

$$
W_{F} \ll N^{1 / 2} L^{-A}
$$

for any $A>0$.

For $k \leq N^{(5 / 48)-\varepsilon}$ and if none of the integers $q \in A_{k}$ is $N$ exceptional, then for $F \in\{C, E\}$

$$
W_{F} \ll N^{1 / 2} L^{-A}
$$

for any $A>0$.

Lemma 4.2. If $k \leq N^{(2 / 15)-\varepsilon}$, then for $F \in\{A, B, C, D, E\}$

$$
I_{F} \ll N L^{M}
$$

for a certain $M>0$.

In the sequel, we will also use the following lemma, which is the estimate (1.1) in [6]: 
Lemma 4.3. Let $N^{*}(\alpha, T, q)$ denote the number of zeros $\sigma+i t$ of all $L$-functions to primitive characters modulo $q$ within the region $\sigma \geq \alpha$, $|t| \leq T$. Then, for a positive integer $m$,

$$
\sum_{\substack{q \leq P \\ m \mid q}} N^{*}(\alpha, T, q) \ll\left(\frac{P^{2} T}{m}\right)^{((12 / 5)+\varepsilon)(1-\alpha)} .
$$

5. Proof of Lemma 4.1 for $W_{A}$. In order to prove the lemma it is enough to show that

$$
W_{A, R} \ll N^{1 / 2}\left(\frac{R}{k}\right)^{1 / 4} k^{1 / 3} L^{-A},
$$

where

$$
W_{A, R}=\sum_{\substack{r \sim R \\ k \mid r}} \sum_{(\bmod r)} *\left(\int_{-k / r Q}^{k / r Q}|W(\lambda, \chi)|^{2} d \lambda\right)^{1 / 2}
$$

for $R \leq P_{1} k / 2$. Applying Lemma 1, [4], we see

$$
\int_{-k / r Q}^{k / r Q}|W(\lambda, \chi)|^{2} d \lambda
$$

$$
\ll(Q R / k)^{-2} \int_{N / 8}^{N}\left|\sum_{\substack{t<m \leq t+Q r / k \\ N / 4<m \leq N}} \Lambda(m) \chi(m)-E_{0}(\chi) \sum_{\substack{t<m \leq t+Q r / k \\ N / 4<m \leq N}} 1\right|^{2} d t .
$$

We note that $E_{0}(\chi)=0$ because of $R \geq k$ and the primitivity of the characters. We set $X=\max (N / 4, t)$ and $X+Y=\min (N, t+Q r / k)$. We apply a slight modification of Heath-Brown's identity [5]

$-\frac{\zeta^{\prime}}{\zeta}(s)=\sum_{j=1}^{K}\left(\begin{array}{c}K \\ j\end{array}\right)(-1)^{j-1} \zeta^{\prime}(s) \zeta^{j-1}(s) M^{j}(s)-\frac{\zeta^{\prime}}{\zeta}(s)(1-\zeta(s) M(s))^{K}$,

with $K=5$ and

$$
M(s)=\sum_{n \leq N^{1 / 5}} \mu(n) n^{-s}
$$


to the sum

$$
\sum_{X<m \leq X+Y} \Lambda(m) \chi(m)
$$

Arguing exactly as in part III, [19] we find by applying Heath-Brown's identity and Perron's summation formula that the inner sum of (5.2) is a linear combination of $O\left(L^{c}\right)$ terms of the form

$$
\begin{aligned}
& S_{I_{a_{1}}, \ldots, I_{a_{10}}} \\
& =\frac{1}{2 \pi i} \int_{-T}^{T} F\left(\frac{1}{2}+i u, \chi\right) \frac{(X+Y)^{((1 / 2)+i u)}-X^{((1 / 2)+i u)}}{(1 / 2)+i u} d u \\
& \quad+O\left(T^{-1} N L^{2}\right),
\end{aligned}
$$

where $2 \leq T \leq N$,

$$
\begin{gathered}
F(s, \chi)=\prod_{j=1}^{10} f_{j}(s, \chi), \quad f_{j}(s, \chi)=\sum_{n \in I_{j}} a_{j}(n) \chi(n) n^{-s}, \\
a_{j}(n)= \begin{cases}\log n \text { or } 1 \quad j=1, \\
1 \quad & 1<j \leq 5, \quad I_{j}=\left(N_{j}, 2 N_{j}\right], \quad 1 \leq j \leq 10, \\
\mu(n) & 6 \leq j \leq 10 .\end{cases} \\
\quad N \ll \prod_{j=1}^{10} N_{j} \ll N, \quad N_{j} \leq N^{1 / 5}, \quad 6 \leq j \leq 10 .
\end{gathered}
$$

Since

$$
\frac{(X+Y)^{((1 / 2)+i u)}-X^{((1 / 2)+i u)}}{(1 / 2)+i u} \ll \min \left(Q R k^{-1} N^{-1 / 2}, N^{1 / 2}(|u|+1)^{-1}\right)
$$

by taking $T=N$ and $T_{0}=N(Q R / k)^{-1}$, we conclude that, for a sufficiently large $G=G(M), S_{I_{a_{1}}, \ldots, I_{a_{10}}}$ is bounded by

$$
\begin{aligned}
\ll & Q R k^{-1} N^{-1 / 2} \int_{-T_{0}}^{T_{0}}\left|F\left(\frac{1}{2}+i u, \chi\right)\right| d u \\
& +N^{1 / 2} \int_{T_{0} \leq|u| \leq T}\left|F\left(\frac{1}{2}+i u, \chi\right)\right| \frac{d u}{|u|}+L^{2},
\end{aligned}
$$


Thus we derive from (5.2) that, in order to prove (5.1), it is enough to show that, for $R \leq P_{1} k / 2$,

$$
\sum_{\substack{r \sim R \\ k \mid r}} \sum_{\chi}^{*} \int_{0}^{T_{0}}\left|F\left(\frac{1}{2}+i t, \chi\right)\right| d t \ll N^{1 / 2} R^{1 / 4} k^{1 / 12} L^{-A}
$$

$$
\begin{gathered}
\sum_{\substack{r \sim R \\
k \mid r}} \sum_{T_{1}}^{*} \int^{2 T_{1}}\left|F\left(\frac{1}{2}+i t, \chi\right)\right| d t \ll N^{-1 / 2} Q R^{5 / 4} k^{-1 / 12} T_{1} L^{-A}, \\
T_{0}<\left|T_{1}\right| \leq T
\end{gathered}
$$

The inequalities (5.4) and (5.5) are both derived from the following lemma which is shown for $m=1$ in Lemma 5.2, [10] and for the general case $m \geq 1$ in Lemma 2.1 in $[8]$.

Lemma 5.1. Let $F(s, \chi)$ be defined as above. Then, for any $R \geq 1$ and $T_{2}>0$,

$$
\begin{aligned}
\sum_{\substack{r \sim R \\
m \mid r}} \sum_{\chi}^{*} \int_{T_{2}}^{2 T_{2}} \mid F & \left(\frac{1}{2}+i t, \chi\right) \mid d t \\
& \ll\left(\frac{R^{2}}{m} T_{2}+\frac{R}{m^{1 / 2}} T_{2}^{1 / 2} N^{3 / 10}+N^{1 / 2}\right) L^{c}
\end{aligned}
$$

Using (2.1) and (2.4), the estimates (5.4) and (5.5) follow from Lemma 5.1 by setting $T_{2}=T_{0}$ and $T_{2}=T_{1}$, respectively, provided that $k \leq N^{2 / 15-\varepsilon}$ and $H$ is chosen sufficiently large in (2.1).

6. Proof of Lemma $\mathbf{4 . 2}$ for $I_{A}$. To prove the lemma it is enough to show that

$$
\max _{R \leq P_{1} k / 2} \sum_{\substack{r \sim R \\ k \mid r}} \sum_{(\bmod r)}{ }^{*} \max _{|\lambda| \leq k / r Q}|W(\lambda, \chi)| \ll N k^{1 / 3} L^{c} .
$$


Arguing as in the section before (we do not have to apply Gallagher's lemma here) we find

$W(\lambda, \chi)$

$$
\begin{aligned}
& \ll L^{c} \max _{I_{a_{1}}, \ldots, I_{a_{10}}}\left|\int_{-T}^{T} F\left(\frac{1}{2}+i t, \chi\right) d t \int_{N / 4}^{N} u^{-1 / 2} e\left(\frac{t}{2 \pi} \log u+\lambda u\right) d u\right| \\
& \quad+L^{2} k^{3} .
\end{aligned}
$$

Here, we have set $T=N$ and used that $|\lambda| \leq k / Q$. Estimating the inner integral by Lemma 3.1 we obtain

$$
\begin{aligned}
\mid \int_{N / 4}^{N} u^{-1 / 2} e & \left(\frac{t}{2 \pi} \log u+l u\right) d u \mid \\
& \ll N^{-1 / 2} \min \left(\frac{N}{\sqrt{|t|+1}}, \frac{N}{\min _{N / 2<u \leq N}|t+2 \pi \lambda u|}\right) .
\end{aligned}
$$

Taking $T_{0}=4 \pi N(Q R / k)^{-1}$ we conclude that in order to prove the lemma it is enough to prove that

$$
\begin{aligned}
& \sum_{\substack{r \sim R \\
k \mid r}} \sum_{\chi}^{*} \int_{0}^{T_{0}}\left|F\left(\frac{1}{2}+i t, \chi\right)\right| d t \ll N^{1 / 2} T_{0}^{1 / 2} k^{1 / 3} L^{c}, \\
& \sum_{\substack{r \sim R \\
k \mid r}} \sum_{\chi}^{*} \int_{T_{1}}^{2 T_{1}}\left|F\left(\frac{1}{2}+i t, \chi\right)\right| d t \ll N^{1 / 2} k^{1 / 3} T_{1} L^{c}, \quad T_{0}<\left|T_{1}\right| \leq T .
\end{aligned}
$$

These estimates follow from Lemma 5.1 for $k \leq N^{2 / 15-\varepsilon}$.

7. Proof of Lemma 4.1 for $\mathbf{W}_{B}, \mathbf{W}_{C}, \mathbf{W}_{D}$ and $\mathbf{W}_{E}$. Arguing analogously to Section 5, we find that the proof of Lemma 4.1 for $F=B$ reduces to the proof of the following two estimates: For $T=N$, $T_{0}=N(Q R)^{-1}, R \leq P_{1} / 2$ and $k \leq N^{3 / 16-\varepsilon}$, there must hold

$$
\sum_{r \sim R} \sum_{\chi}^{*} \int_{0}^{T_{0}}\left|F\left(\frac{1}{2}+i t, \chi\right)\right| d t \ll N^{1 / 2} R^{1 / 4} k^{1 / 3} L^{-A},
$$

$$
\begin{gathered}
\sum_{r \sim R} \sum_{\chi}^{*} \int_{T_{1}}^{2 T_{1}}\left|F\left(\frac{1}{2}+i t, \chi\right)\right| d t \ll N^{-1 / 2} Q R^{5 / 4} k^{1 / 3} T_{1} L^{-A}, \\
T_{0}<\left|T_{1}\right| \leq T
\end{gathered}
$$


The estimates (7.1) and (7.2) follow from (2.1) and Lemma 5.1. For the case $F=C$, we treat separately the cases $R / k \leq L^{V}$ and $R / k \geq L^{V}$ for a sufficiently large $V$ to be determined later. In the second case, it is enough to show, using Lemma 5.1, that for $T=N, T_{0}=N(Q R)^{-1}$, $R \leq P_{2} / 2$, and $k \leq N^{3 / 20-\varepsilon}$, we have

$$
\sum_{\substack{r \sim R \\ k \mid r}} \sum_{\chi}^{*} \int_{0}^{T_{0}}\left|F\left(\frac{1}{2}+i t, \chi\right)\right| d t \ll N^{1 / 2}\left(\frac{R}{k}\right)^{1 / 6} L^{-A}
$$

$$
\begin{gathered}
\sum_{\substack{r \sim R \\
k \mid r}} \sum_{\chi}^{*} \int_{T_{1}}^{2 T_{1}}\left|F\left(\frac{1}{2}+i t, \chi\right)\right| d t \ll N^{-1 / 2} Q R^{7 / 6} k^{-1 / 6} T_{1} L^{-A} \\
T_{0}<\left|T_{1}\right| \leq T
\end{gathered}
$$

In the case $R / k \leq L^{V}$, we can estimate the sum on the righthand side of (5.2) by using the zero expansion of the von Mangoldt-function:

$$
\begin{aligned}
\sum_{\substack{t<m \leq t+Q r \\
N / 4<m \leq N}} \Lambda(m) \chi(m)-E_{0}(\chi) \sum_{\substack{t<m \leq t+Q r \\
N / 4<m \leq N}} 1 \\
=\sum_{X<m \leq X+Y} \Lambda(m) \chi(m)-E_{0}(\chi) \sum_{X<m \leq X+Y} 1 \\
\ll \sum_{|\operatorname{Im} \rho| \leq T_{3}}\left|\frac{(X+Y)^{\rho}}{\rho}-\frac{X^{\rho}}{\rho}\right|+O\left(\frac{N}{T_{3}} L^{2}\right) \\
\ll Q R \sum_{|\operatorname{Im} \rho| \leq T_{3}} N^{\beta-1}+O\left(\frac{N}{T_{3}} L^{2}\right),
\end{aligned}
$$

where $\rho$ runs over the nontrivial zeros of the $L$-function corresponding to $\chi \bmod r$ with $|\operatorname{Im} \rho| \leq T_{3}$ and $\beta=\operatorname{Re} \rho$. Arguing as in (5.2), we see from (7.5) for $T_{3}=k^{2} L^{2} V$ that

$$
\begin{aligned}
\int_{1 / r Q}^{1 / r Q}|W(\lambda, \chi)|^{2} d \lambda & \\
& \ll N\left(\sum_{|\operatorname{Im} \rho| \leq k^{2} L^{2 V}} N^{\beta-1}\right)^{2}+O\left((Q r)^{-2} N^{3} k^{-4} L^{4-4 V}\right) .
\end{aligned}
$$


Using (1.2) and defining $W_{C, R}$ analogously to (5.1), we use the assumptions of Theorem 2 and Lemma 4.3 and obtain for $k \leq N^{5 / 36-\varepsilon}$

$$
\begin{aligned}
W_{C, R} & \ll N^{1 / 2} \sum_{\substack{r \leq k L^{V} \\
k \mid r}} \sum_{\chi \bmod r} * \sum_{|\operatorname{Im} \rho| \leq k^{2} L^{2 V}} N^{\beta-1}+N^{1 / 2} L^{-A} \\
& \ll N^{1 / 2} L^{C} \max _{1 / 2 \leq \beta \leq 1-E L_{2} / L}\left(N^{((5 / 12)-2 \varepsilon)((12 / 5)+\varepsilon)(1-\beta)} N^{\beta-1}\right) \\
& +N^{1 / 2} L^{-A} \\
& \ll N^{1 / 2} L^{-A},
\end{aligned}
$$

for a sufficiently large $E=E(A, \varepsilon)$. In the case $F=D$, we distinguish between the cases $R>L^{W}$ for a sufficiently large $W$ to be determined later and $R \leq L^{W}$. In the first case, we argue as in Section 4 and see that it is enough to show, using Lemma 5.1, the following. If $T=N$ and $T_{0}=N(Q R)^{-1}, r \leq P_{2} / 2 k$ and $k \leq N^{4 / 25-\varepsilon}$, then:

$$
\begin{gathered}
\sum_{r \sim R} \sum_{\chi} * \int_{0}^{T_{0}}\left|F\left(\frac{1}{2}+i t, \chi\right)\right| d t \ll N^{1 / 2} R^{1 / 4} L^{-A}, \\
\sum_{r \sim R} \sum_{\chi}^{*} \int_{T_{1}}^{2 T_{1}}\left|F\left(\frac{1}{2}+i t, \chi\right)\right| d t \ll N^{-1 / 2} Q R^{5 / 4} T_{1} L^{-A}, \\
T_{0}<\left|T_{1}\right| \leq T .
\end{gathered}
$$

If $R \leq L^{W}$, we apply Lemma 4.3 and the fact that $L(\sigma+i t, \chi)$ with $\chi \bmod r$ and $r \leq L^{D}$ has no zeros in the region, see [15, VIII Satz 6.2)

$$
\sigma \geq 1-\delta(T):=1-\frac{c_{0}}{\log r+(\log (T+2))^{4 / 5}}, \quad|t| \leq T,
$$

where $c_{0}$ is an absolute constant. Taking $T=N^{1 / 3}$ and $k \leq N^{3 / 20-\varepsilon}$, we obtain from Lemma 4.3 from (7.5)

$$
\begin{aligned}
& \int_{-1 / Q r}^{1 / Q r}|W(\lambda, \chi)|^{2} d \lambda \ll N\left(\sum_{|\operatorname{Im} \rho| \leq N^{1 / 3}} N^{\beta-1}\right)^{2}+(Q r)^{-2} N^{1+(4 / 3)} L^{4} \\
& \ll N L^{c}\left(\max _{(1 / 2) \leq \beta \leq 1-\delta(T)} N^{((4 / 5)+\varepsilon)(1-\beta)} N^{(\beta-1)}\right)^{2}+N^{1 / 3} k^{4} L^{2 W+4} \\
& \ll N \exp \left(-c L^{1 / 5}\right) .
\end{aligned}
$$


This proves the lemma for $R \leq L^{W}$. For the case $F=E$, we treat separately the cases $R / k^{2} \leq L^{V}$ and $R / k^{2} \geq L^{V}$ for a sufficiently large $V$ to be determined later. In the second case, it is enough to show, using Lemma 5.1, that for $T=N, T_{0}=N(Q R)^{-1}, R \leq P_{2} / 2$ and $k \leq N^{5 / 48-\varepsilon}$, we have

$$
\begin{gathered}
\sum_{\substack{r \sim R \\
k^{2} \mid r}} \sum_{\chi}^{*} \int_{0}^{T_{0}}\left|F\left(\frac{1}{2}+i t, \chi\right)\right| d t \ll N^{1 / 2}\left(\frac{R}{k^{2}}\right)^{1 / 6} L^{-A}, \\
\sum_{\substack{r \sim R \\
k^{2} \mid r}} \sum^{*} \int_{T_{1}}^{2 T_{1}}\left|F\left(\frac{1}{2}+i t, \chi\right)\right| d t \ll N^{-1 / 2} Q R^{7 / 6} k^{-1 / 3} T_{1} L^{-A}, \\
T_{0}<\left|T_{1}\right| \leq T .
\end{gathered}
$$

For $R / k^{2} \leq L^{V}$, we argue as in (7.6).

8. Proof of Lemma 4.2 for $\mathbf{I}_{B}, \mathbf{I}_{C}, \mathbf{I}_{D}$, and $\mathbf{I}_{E}$. Throughout this section we set $T=N$ and $T_{0}=N(Q R)^{-1}$. Arguing as in Section 6 , we see that to estimate $I_{B}$ it is enough to show that for $k \leq N^{3 / 16-\varepsilon}$ and $R \leq P_{1} / 2$, we have

$$
\begin{gathered}
\sum_{r \sim R} \sum_{\chi}^{*} \int_{0}^{T_{0}}\left|F\left(\frac{1}{2}+i t, \chi\right)\right| d t \ll N^{1 / 2}\left(T_{0}+1\right)^{1 / 2} k^{1 / 3} L^{c}, \\
\sum_{r \sim R} \sum_{\chi}^{*} \int_{T_{1}}^{2 T_{1}}\left|F\left(\frac{1}{2}+i t, \chi\right)\right| d t \ll N^{1 / 2} k^{1 / 3} T_{1} L^{c}, \\
T_{0}<\left|T_{1}\right| \leq T .
\end{gathered}
$$

For the estimate of $I_{C}$ it is enough to show that, for $k \leq N^{3 / 20-\varepsilon}$ and $R \leq P_{2} / 2$, we have:

$$
\begin{gathered}
\sum_{\substack{r \sim R \\
k \mid r}} \sum_{\chi}^{*} \int_{0}^{T_{0}}\left|F\left(\frac{1}{2}+i t, \chi\right)\right| d t \ll N^{1 / 2}\left(T_{0}+1\right)^{1 / 2}\left(\frac{R}{k}\right)^{1 / 6} L^{c}, \\
\sum_{\substack{r \sim R \\
k \mid r}} \sum_{\chi}^{*} \int_{T_{1}}^{2 T_{1}}\left|F\left(\frac{1}{2}+i t, \chi\right)\right| d t \ll N^{1 / 2}\left(\frac{R}{k}\right)^{1 / 6} T_{1} L^{c}, \\
T_{0}<\left|T_{1}\right| \leq T .
\end{gathered}
$$


These estimates follow from Lemma 5.1.

For the estimate of $I_{D}$ it is enough to show that if $k \leq N^{1 / 5-\varepsilon}$ and $R \leq P_{2} / 2 k$, then

$$
\begin{gathered}
\sum_{r \sim R} \sum_{\chi}^{*} \int_{0}^{T_{0}}\left|F\left(\frac{1}{2}+i t, \chi\right)\right| d t \ll N^{1 / 2}\left(T_{0}+1\right)^{1 / 2} L^{c}, \\
\sum_{r \sim R} \sum_{\chi}^{*} \int_{T_{1}}^{2 T_{1}}\left|F\left(\frac{1}{2}+i t, \chi\right)\right| d t \ll N^{1 / 2} T_{1} L^{c}, \\
T_{0}<\left|T_{1}\right| \leq T .
\end{gathered}
$$

These estimates follow from Lemma 5.1.

Likewise, for the proof of the estimate for $I_{E}$, we use Lemma 5.1 to show that for the estimate of $I_{C}$ it is enough to show that for $k \leq N^{3 / 20-\varepsilon}$ and $R \leq P_{2} / 2$, we have:

$$
\begin{gathered}
\sum_{\substack{r \sim R \\
k^{2} \mid r}} \sum_{\chi}^{*} \int_{0}^{T_{0}}\left|F\left(\frac{1}{2}+i t, \chi\right)\right| d t \ll N^{1 / 2}\left(\frac{R}{k^{2}}\right)^{1 / 6}\left(T_{0}+1\right)^{1 / 2} L^{c}, \\
\sum_{\substack{r \sim R \\
k^{2} \mid r}} \sum_{\chi}^{*} \int_{T_{1}}^{2 T_{1}}\left|F\left(\frac{1}{2}+i t, \chi\right)\right| d t \ll N^{1 / 2}\left(\frac{R}{k^{2}}\right)^{1 / 6} T_{1} L^{c}, \\
T_{0}<\left|T_{1}\right| \leq T .
\end{gathered}
$$

9. Proof of Theorem 3. Using (1.2) and Lemma 4.3, we derive an estimate for the number of the $N$-exceptional zeros. We find

$$
\sum_{q \leq N} N^{*}\left(1-\frac{E L_{2}}{L}, q\right) \ll N^{((36 / 5)+\varepsilon)\left(E L_{2} / L\right)} \ll L^{36 E / 5+\varepsilon} .
$$

Thus, there do not exist more than $\ll L^{36 E / 5+\varepsilon} N$-exceptional integers. Each integer $\leq N$ has at most $O(\log N)$ different prime factors. Thus, each $N$-exceptional integer does belong to at most $O(\log N)$ different sets $A_{k}$. Therefore, there are no more than $O\left(L^{36 E / 5+1+\varepsilon}\right)$ prime numbers $k, 1 \leq k \leq N$, such that at least one of the integers $q \in A_{k}$ is $N$-exceptional. 


\section{REFERENCES}

1. R. Ayoub, On Rademacher's extension of the Goldbach-Vinogradov theorem, Canad. J. Math. (1953), 482-491.

2. C. Bauer, On Goldbach's conjecture in arithmetic progressions, Stud. Sci. Math. Hungar. 37 (2001), 1-20.

3. - On the exceptional set for the sum of a prime and the $k$-th power of a prime, Stud. Sci. Math. 35 (1999), 291-330.

4. P.X. Gallagher, A large sieve density estimate near $\sigma=1$, Invent. Math. 11 (1970), 329-339.

5. D.R. Heath-Brown, Prime numbers in short intervals and a generalized Vaughan's identity, Canad. J. Math. 34 (1982), 1365-1377.

6. N.M. Huxley, Large values of Dirichlet polynomials III, Acta Arith. 26 (1975), 435-444.

7. Jianya Liu, The Goldbach-Vinogradov theorem with three primes in a thin subset, Chinese Annals Math. 19 (1998), 479-488.

8. J.Y. Liu, On Lagrange's theorem with prime variables, Quart. J. Math. 54 December (2003), 453-462.

9. J.Y. Liu and T. Zhan, The ternary Goldbach problem in arithmetic progressions, Acta Arith. 82 (1997), 197-227.

10. J.Y. Liu and M.C. Liu, The exceptional set in the four prime squares problem, Illinois J. Math. 44 (2000), 272-293.

11. M.C. Liu and T. Zhan, The Goldbach problem with primes in arithmetic progressions, in Analytic number theory (Yoichi Motohashi, ed.), London Math. Soc. Lecture Note Ser., vol. 247, Cambridge Univ. Press, Cambridge, 1997, pp. 227-251.

12. H.L. Montgomery and R.C. Vaughan, On the exceptional set in Goldbach's problem, Acta Arith. 27 (1975), 353-370.

13. Chengdong Pan and Chengbiao Pan, Analytic number theory, Science Press, Beijing, 1992 (in Chinese).

14. — Goldbach conjecture, Science Press, Beijing, 1991 (in English).

15. K. Prachar, Primzahlverteilung, Springer Verlag, Berlin, 1978.

16. H.A. Rademacher, Ueber eine Erweiterung des Goldbachschen problems, Math. Z. 25 (1926).

17. I.M. Vinogradov, Representation of an odd number as the sum of three primes, Dokl. Akad. Nauk. SSSR 15 (1937), 291-294.

18. A. Zaccagnini, The exceptional set for the sum of a prime and a $k$-th power, Mathematika 39 (1992), 400-421

19. T. Zhan, On the representation of a large odd integer as a sum of three almost equal primes, Acta Math. Sinica 7 (1991), 259-272. 
Dolby Laboratories, 100 Potrero Avenue, San Francisco, 94103 CA, USA E-mail address: cb@dolby.com

The Department of Mathematics, The Capital Normal University, BeiJING 100037, P.R.CHINA

E-mail address: yhwang@mail.cnu.edu.cn 